\title{
The Impact of Ice Crystal Shapes, Size Distributions, and Spatial Structures of Cirrus Clouds on Solar Radiative Fluxes
}

\author{
I. SChlimme AND A. MACKE \\ Leibniz-Institute for Marine Sciences IFM-GEOMAR, Kiel, Germany \\ J. REICHARDT \\ Meteorologisches Observatorium Lindenberg, Deutscher Wetterdienst, Tauche, Germany
}

(Manuscript received 22 September 2003, in final form 10 August 2004)

\begin{abstract}
The solar radiative properties of cirrus clouds depend on ice particle shape, size, and orientation, as well as on the spatial cloud structure. Radiation schemes in atmospheric circulation models rely on estimates of cloud optical thickness only. In the present work, a Monte Carlo radiative transfer code is applied to various cirrus cloud scenarios to obtain the radiative response of uncertainties in the above-mentioned microphysical and spatial cloud properties (except orientation). First, plane-parallel homogeneous (0D) clouds with different crystal shapes (hexagonal columns, irregular polycrystals) and 114 different size distributions have been considered. The resulting variabilities in the solar radiative fluxes are in the order of a few percent for the reflected and about $1 \%$ for the diffusely transmitted fluxes. Largest variabilities in the order of $10 \%$ to $30 \%$ are found for the solar broadband absorptance. However, these variabilities are smaller than the flux differences caused by the choice of ice particle geometries.

The influence of cloud inhomogeneities on the radiative fluxes has been examined with the help of time series of Raman lidar extinction coefficient profiles as input for the radiative transfer calculations. Significant differences between results for inhomogeneous and plane-parallel clouds were found. These differences are in the same order of magnitude as those arising from using extremely different crystal shapes for the radiative transfer calculations. From this sensitivity study, the ranking of cirrus cloud properties according to their importance in solar broadband radiative transfer is optical thickness, ice crystal shape, ice particle size, and spatial structure.
\end{abstract}

\section{Introduction}

The Intergovernmental Panel on Climate Change (IPCC) Report (Houghton et al. 2001) identifies cloud effects on the radiation balance as an important component in climate change prediction. These effects are subject to many uncertainties due to the extreme variability and complexity of clouds. Especially, ice particles in cirrus clouds exhibit a strong spatial and temporal variability in particle size and shape as shown in numerous results from international field campaigns like the International Cirrus Experiment (ICE), the European Cloud and Radiation Experiment (EUCREX; Raschke 1997) or the First International Satellite Cloud Climatology Project (ISCCP) Regional Experiment (FIRE; Kinne et al. 1997). The situation is further complicated by the fact that the microphysical properties of

Corresponding author address: Andreas Macke, LeibnizInstitute for Marine Sciences, Düsternbrooker Weg 20, 24105 Kiel, Germany.

E-mail: amacke@ifm-geomar.de cirrus clouds are poorly correlated with the actual ambient atmospheric conditions (Korolev et al. 2001) although there is a tendency toward more regular and more pristine particle shapes as temperature and water vapor supply decrease (Magano and Lee 1966).

In general, low and midlevel clouds contribute with a net cooling to the radiation budget of our planet, where the magnitude of this cooling strongly depends on cloud optical thickness and cloud-top height (PoetzschHeffter et al. 1995). On the other hand, cirrus clouds may have a positive cloud radiative forcing (Chen et al. 2000). Furthermore, for the same optical thickness or ice water path, the forcing can switch sign depending on ice crystal geometry and size (Zhang et al. 1999). Thus, in contrast to low and midlevel clouds, the microphysical composition of cirrus clouds is an important parameter in determining the sign of the net cloud radiative forcing. The aim of this study is to quantify the effect of cirrus cloud variabilities on the solar radiative fluxes. Radiative transfer in the thermal is not addressed here mainly because the effect of cloud microphysics on the thermally emitted/transmitted flux is less pronounced 
in this spectral range (Zhang et al. 1999). Furthermore, large uncertainties still exist in the correct treatment of scattering and absorption properties at small particle size parameters (Mishchenko et al. 2000). In particular, no theories exist to predict the scattering and absorption behavior of small and highly nonspherical/ nonhomogenous ice particles.

The present study is composed of two parts. In section 2, the variability in spectrally resolved and in solar broadband radiative fluxes due to varying ice crystal shapes and size distributions is estimated. While the particle shapes are assumed to be either perfectly hexagonal or completely irregular, the size distributions are taken from in situ measurements. This approach corresponds to an error analysis of solar cirrus radiative transfer for the common situation that no information on the microphysical composition is available. This part completes the work by Macke et al. (1998), where the role of shape and size distributions on the single scattering and absorption properties of cirrus clouds was examined. There, it was shown that the effect of varying size distributions is smaller than the effect of using extremely different ice crystal shapes. However, it remains to be seen how these single scattering variabilities affect the multiple scattering properties of entire cirrus clouds.

The second part of the present study (section 3 ) investigates the influence of vertical and horizontal cloud inhomogeneity on the solar broadband fluxes. The twodimensional (2D) cloud structures are taken from Raman lidar measurements. Again, this part can be interpreted as an error analysis for the usual assumption of plane-parallel homogeneous clouds. A brief summary as well as concluding remarks are presented in section 4 .

\section{Variability of microphysical properties}

The natural variability of ice crystal sizes is approximately taken into account by performing the radiative transfer calculations for a large number of observed size distributions. The latter are taken from several flight missions during the 1994 EUCREX campaign (Francis 1995). Each size distribution is a composite of Particle Measurements Systems two-dimensional cloud and precipitation (PMS $2 \mathrm{D}-\mathrm{C}$ and $2 \mathrm{D}-\mathrm{P}$, respectively) probe measurements (20-3000 $\mu \mathrm{m}$ total size range) averaged along horizontal flight legs of roughly $100 \mathrm{~km}$. Phase functions and single scattering albedos have been calculated by means of a ray-tracing technique for hexagonal ice columns and fractal polycrystals (Macke et al. 1996). The particle sizes and geometries are the same as in Macke et al. (1998) but more spectral intervals have been taken into account (as described below) to cover the complete solar spectral range. The 114 size distributions used possess an average effective radius of $169 \mu \mathrm{m}$ and a standard deviation of $102 \mu \mathrm{m}$. The term radius refers to the radius of a projected area equivalent sphere. The effective radius is defined as the ratio of the third moment of the size distribution to the second moment (Hansen and Travis 1974). A detailed description of the size distribution statistics and the distribution-averaged single scattering and absorption properties are given in Macke et al. (1998).

The spectrally resolved and solar broadband irradiances, that is, reflected, transmitted, and absorbed fluxes, have been calculated by means of the third Monte Carlo radiative transfer model GRIMALDI (Scheirer and Macke 2001, 2003). The Monte Carlo algorithm traces scattering and absorption processes of a sufficiently large number of solar photons through a predefined cloud structure. The latter is described by a gridded spatial distribution of scattering phase function, single scattering albedo, and volume extinction coefficient. See Macke et al. (1999) for a description of the Monte Carlo scheme. A Monte Carlo model exactly simulates the radiative transfer processes, and its accuracy is limited only by the number of photons used in the simulations. In the present study, the number of one million photons per model case is chosen, which guarantees a statistical error smaller than $1 \%$.

Radiative transfer calculations have been performed for plane-parallel homogeneous cirrus clouds with optical thickness values $0.5,1,2,4,8$, and 12 , for solar zenith angles $35^{\circ}$ and $65^{\circ}$, and for 13 spectral intervals that cover the solar spectrum (see Table 1). Cloud optical thickness is assumed to be independent of the wavelength. This is justified by the use of large ice crystals (size parameter much larger than 1 ), and by the size distribution averaging. The cirrus clouds are embedded in a U.S. standard atmosphere. Owing to the origin of the data, the midlatitude summer atmosphere has been selected for the size distribution sensitivity study, and the subarctic winter atmosphere for the lidar case study. The surface is assumed to be black over the entire spectral range for all scenarios. Reflected flux is computed at the highest $z$ level of the cloud domain, transmitted flux at the bottom of the model domain and absorbed flux applies to the entire vertical column of the model domain from the ground to a height of $12 \mathrm{~km}$.

TABLE 1. Spectral spreading of solar wavelength, spectral flux, and weightings (Thekaekara 1974).

\begin{tabular}{cccc}
\hline $\begin{array}{c}\text { Wavelength } \\
(\mathrm{nm})\end{array}$ & $\begin{array}{c}\text { Wavelength } \\
\text { interval }(\mathrm{nm})\end{array}$ & $\begin{array}{c}\text { Radiant flux } \\
\text { density }\left(\mathrm{W} \mathrm{m}{ }^{-2}\right)\end{array}$ & Weighting \\
\hline 0.308 & $0.2-0.3927$ & 98.2 & 0.072 \\
0.55 & $0.3927-0.72$ & 567.2 & 0.417 \\
0.89 & $0.72-1.185$ & 402.9 & 0.296 \\
1.48 & $1.185-1.7335$ & 178.1 & 0.131 \\
1.78 & $1.7335-1.9659$ & 36.0 & 0.026 \\
2.00 & $1.9659-2.21294$ & 24.9 & 0.018 \\
2.36 & $2.21294-2.54$ & 20.6 & 0.015 \\
2.72 & $2.54-2.79$ & 10.4 & 0.008 \\
2.92 & $2.79-2.9725$ & 5.6 & 0.004 \\
3.07 & $2.9725-3.145$ & 4.2 & 0.003 \\
3.22 & $3.145-3.31$ & 3.3 & 0.002 \\
3.40 & $3.31-3.64505$ & 5.1 & 0.004 \\
3.69 & $3.64505-4.00$ & 3.8 & 0.003 \\
\hline
\end{tabular}


Radiative transfer results for two spectral intervals with center wavelengths of 0.55 and $1.48 \mu \mathrm{m}$ will be discussed in more detail. The first spectral interval represents an almost nonabsorbing case where the maximum of the solar irradiation is also located. The second interval in the near-infrared spectrum represents a moderately absorbing case where a significant amount of solar radiation is still available. Figures 1 and 2 show the solar fluxes at these intervals at $35^{\circ}$ solar zenith angle as a function of cloud optical thickness. Each dot corresponds to one of the 114 calculations, that is, to one of the size distributions, assuming hexagonal ice columns. Averages over all size distributions are shown as large symbols, and are used for the fitting curves (solid lines). For comparison reasons, the fitting curves for fractal particles are shown in dashed lines. Note that the results for absorbed flux have a different ordinate scale at a $0.55-\mu \mathrm{m}$ wavelength.

As was shown by Macke et al. (1998), irregular crystals have smaller asymmetry parameters (mean cosine of the scattering phase function) at moderately and nonabsorbing wavelengths compared to size-equivalent hexagonal symmetric particles, that is, smaller forward scattering. This explains the larger reflected flux (upper left diagrams of Figs. 1 and 2) and the smaller total transmitted flux (upper right diagrams) for this particle type. The total transmitted flux is split into a direct and a diffuse component in the lower right diagrams. The direct transmitted flux decreases exponentially with optical thickness independently of the specific size distribution owing to Beer-Lambert-Bouguer's law of attenuation only. Contrarily, the diffuse transmitted flux reaches a maximum at optical thickness values around 2 , and decreases for larger $\tau$ values due to the strong increase in the reflected flux. Because the diffuse transmitted flux depends on the scattering characteristics of the ice particles it shows a dependence on the choice of the size distribution. Finally, absorbed flux is shown in the lower left diagrams. The extremely small absorptance at the visible wavelength is shown for reasons of completeness only. Because of their more compact and irregular shape fractal particles are stronger reflecting and stronger absorbing than hexagonal symmetric particles. See also Macke et al. (1996, 1998).

In general, the dependence of the radiative fluxes on the size distribution is more pronounced at the absorbing wavelengths simply because larger particles absorb more radiation whereas changes in the scattering characteristics with particle size are small. The spectral signature of the solar constant shifts the region of largest size dependency of the radiative fluxes more toward the smaller wavelengths. This is illustrated in Fig. 3 where
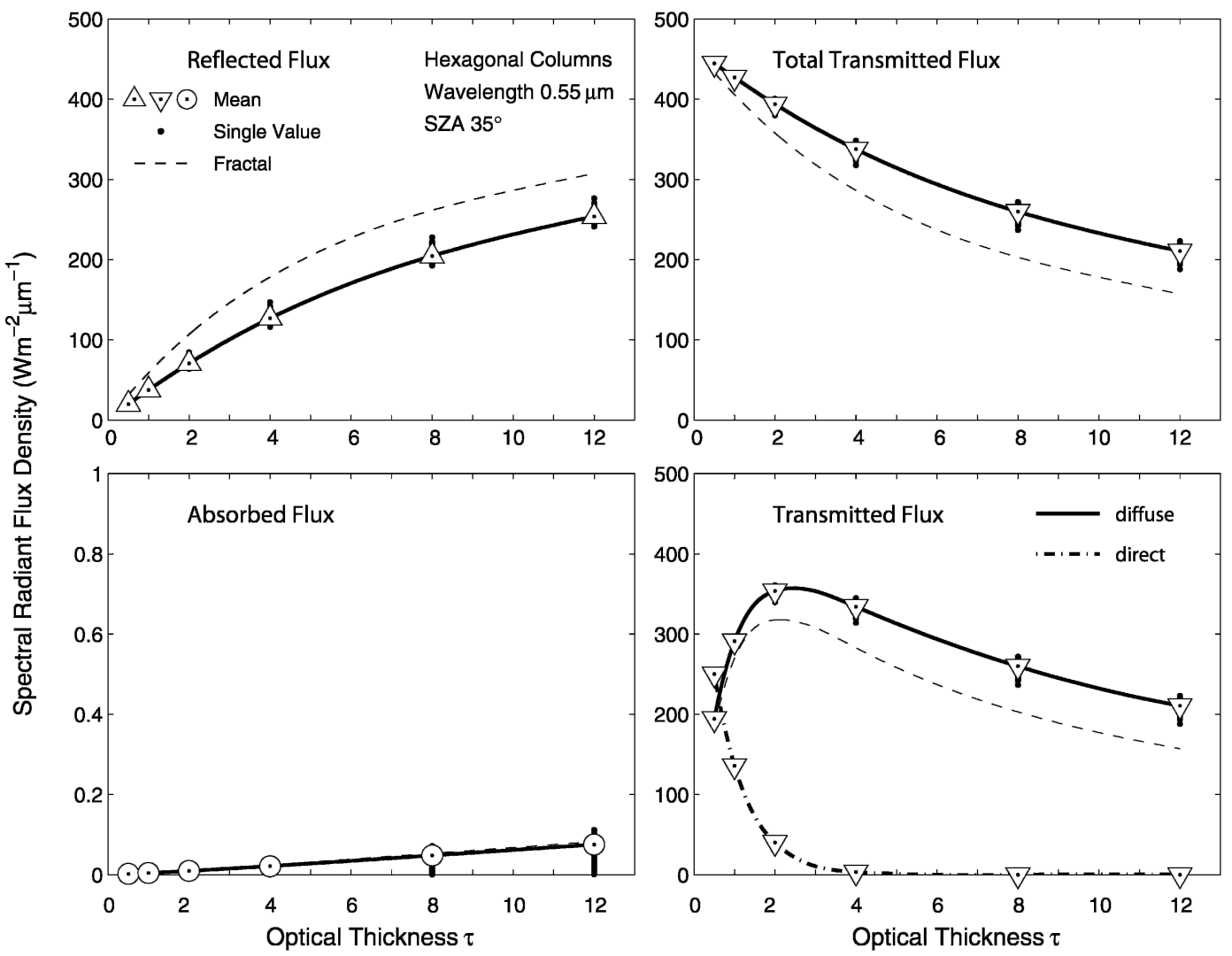

FIG. 1. Spectral fluxes as a function of optical thickness for a wavelength of $0.55 \mu \mathrm{m}$ and a solar zenith angle (SZA) of $35^{\circ}$ for hexagonal columns. The dashed lines show the results for fractal particles. 

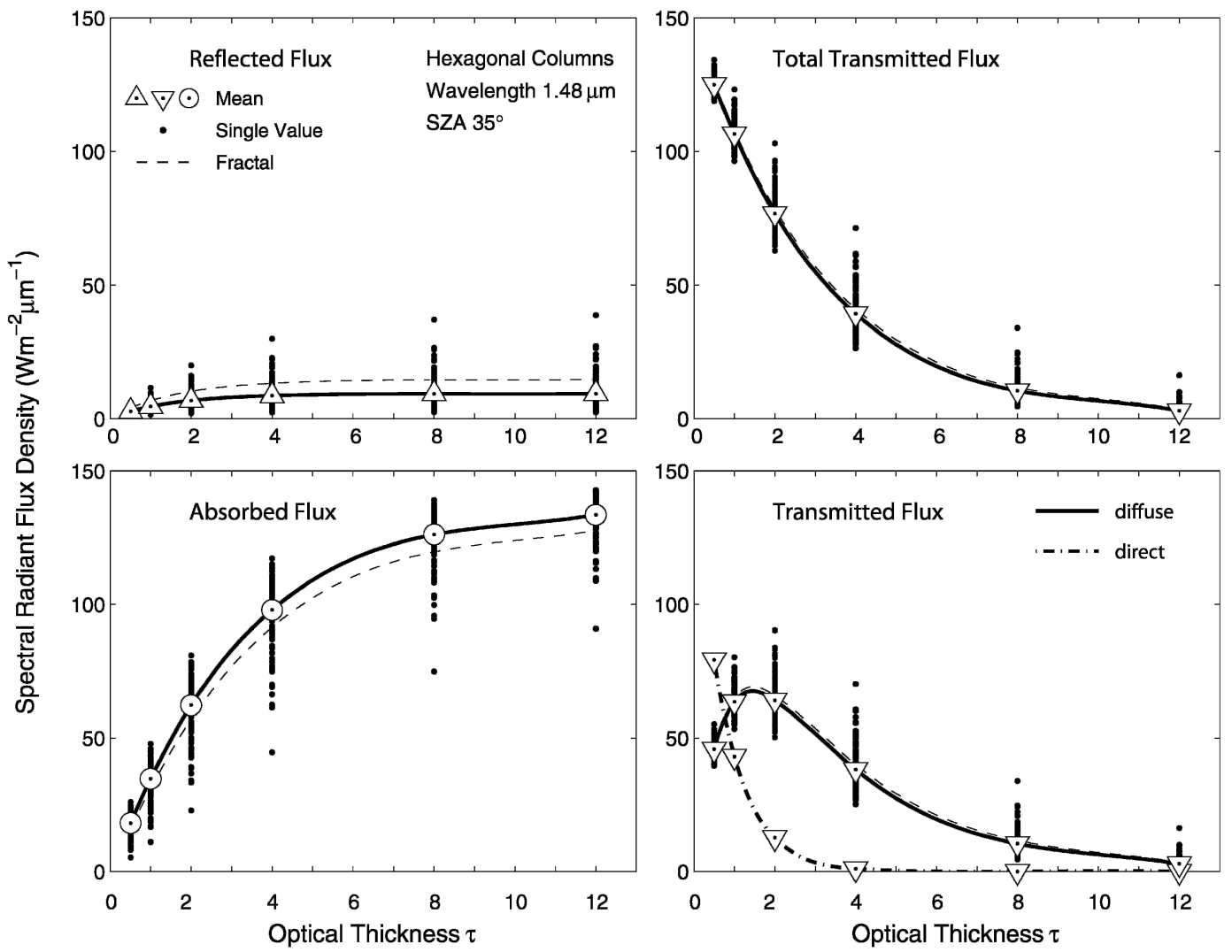

FIG. 2. Same as in Fig. 1 except a function of optical thickness for a wavelength of $1.48 \mu \mathrm{m}$ and an SZA of $35^{\circ}$.

both the size distribution-averaged spectral fluxes and the variation coefficient $(v)$ are shown as functions of wavelength and cloud optical thickness. The variation coefficient $(v)$ is defined as the percentage ratio of flux standard deviation $s$ to flux average value $(\bar{a})$; it is a measure of the influence of the choice of size distributions. Largest variation coefficients are $80 \%$ for the reflected flux at a $2.5-\mu \mathrm{m}$ wavelength, $150 \%$ for the total transmitted flux at the same wavelength, and $30 \%$ for the absorbed flux at visible wavelengths. For the reflected and total transmitted flux, the variation coefficient increases with optical depth due to the increasing number of scattering events. This is because each additional scattering event allows for a wider range of possible ray paths. Only for absorbed flux, the variation coefficient decreases because the absorbed flux quickly reaches saturation and becomes insensitive to the choice of particle size. Here, the energy of the incoming photons is absorbed during the first scattering/absorption events and higher-order events that result from larger optical thicknesses have little influence on the total amount of absorbed energy.

Broadband fluxes and variation coefficients have been calculated by adding the spectral parameters discussed above weighted with the spectral solar constants listed in Table 1. The reason for the unusual band selection in Table 1 is to better resolve spectral regions with strong gradients in the absorbtivity of ice, weighted by the spectral solar constant because the spectral change in light scattering and absorption properties of ice particles is mainly driven by absorptivity of ice.

The radiative transfer results are presented for fractal particles at a solar zenith angle of $35^{\circ}$ (Table 2) and $65^{\circ}$ (Table 3), and for hexagonal columns at $35^{\circ}$ (Table 4 ) and $65^{\circ}$ (Table 5). As explained in the previous paragraph, largest variation coefficients and standard deviations are found for the absorbed flux. Note that the standard variations can get as large as $\sim 32 \mathrm{~W} \mathrm{~m}^{-2}$ here. We select the following two cases as representative for two commonly observed cirrus cloud types. A typical midlatitude cirrus resulting from large-scale frontal lifting may be characterized by hexagonal ice columns, an optical thickness of about 2 , and a solar zenith angle of $65^{\circ}$. A typical tropical anvil cirrus resulting from deep convection shall be characterized by the fractal ice particles, optical thickness 8 , and a solar zenith angle of $35^{\circ}$. For the midlatitude cirrus we find a standard deviation of $10 \mathrm{~W} \mathrm{~m}^{-2}$ for the reflected flux, $3 \mathrm{~W} \mathrm{~m}^{-2}$ for the transmitted flux, and $13 \mathrm{~W} \mathrm{~m}^{-2}$ for the absorbed flux. Corresponding values for our tropical scenario are 19,12 , and $32 \mathrm{~W} \mathrm{~m}^{-2}$, respectively. Thus, any lack of knowledge of the size distribution translates in significant uncertainties in the solar broadband radiative 

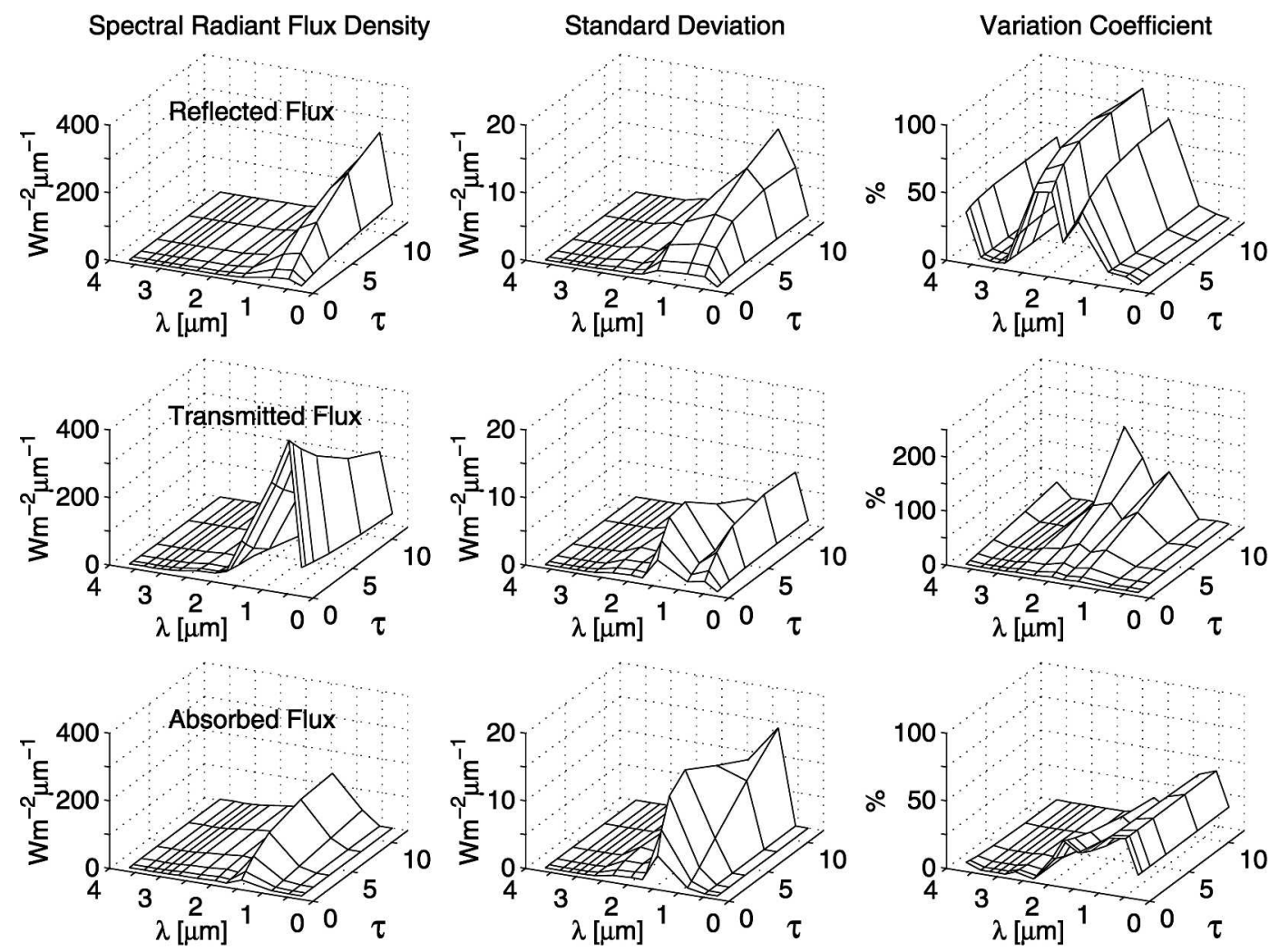

FIG. 3. Spectral fluxes, std dev, and variation coefficients as functions of 13 wavelengths and 5 optical thicknesses for ice columns at an SZA of $35^{\circ}$.

fluxes. However, a comparison with the results for different particle geometries but same optical thickness and solar illumination conditions reveals that the differences in reflected and total transmitted flux due to the two essentially different particle geometries are larger than the standard deviations because of the lack of information regarding the particle sizes. This is in agreement with the findings reported by Macke et al. (1998) for size distribution-averaged single scattering properties. Only the broadband absorbed flux is more sensitive to the size distribution than to the particle shape under consideration. Again, the reasons for this different behavior of reflection and transmission on the one hand, and absorption on the other hand are simply that light scattering characteristics are more shape dependent, whereas absorption mainly depends on the size of the particle.

\section{Variability of cloud structure}

While in the previous section we focused on the variability of cirrus microphysical properties, here we ad-

TABLe 2. Average value $(\bar{a})$, std dev $(s)$, and variation coefficient $(v)$ for broadband reflected, total transmitted, and absorbed fluxes at different optical thicknesses. A fractal crystal shape and a solar zenith angle of $\theta=35^{\circ}$ are used.

\begin{tabular}{|c|c|c|c|c|c|c|c|c|c|c|}
\hline \multirow[b]{4}{*}{$\begin{array}{l}\text { Optical } \\
\text { thickness }\end{array}$} & \multicolumn{10}{|c|}{ Fractal $-\theta=35^{\circ}$} \\
\hline & & & & \multicolumn{4}{|c|}{ Transmitted flux } & & & \\
\hline & \multicolumn{3}{|c|}{ Reflected flux } & \multicolumn{2}{|c|}{ Diffuse } & \multicolumn{2}{|c|}{ Direct } & \multicolumn{3}{|c|}{ Absorbed flux } \\
\hline & \multicolumn{2}{|c|}{$\left(\mathrm{W} \mathrm{m} \mathrm{m}^{-2}\right)$} & $\begin{array}{c}v \\
\%\end{array}$ & \multicolumn{2}{|c|}{$\left(\mathrm{W} \mathrm{m}^{-2}\right)$} & $\begin{array}{l}(v) \\
\%\end{array}$ & $\begin{array}{c}\bar{a} \\
\left(\mathrm{~W} \mathrm{~m}^{-2}\right)\end{array}$ & \multicolumn{2}{|c|}{$\left(\mathrm{W} \mathrm{m} \mathrm{m}^{-2}\right)$} & $\begin{array}{c}\nu \\
\%\end{array}$ \\
\hline 0.5 & 65.3 & 2.4 & 3.6 & 418.6 & 5.4 & 1.2 & 600.3 & 30.1 & 7.7 & 25.6 \\
\hline 1.0 & 120.8 & 4.6 & 3.8 & 610.0 & 9.3 & 1.5 & 326.0 & 57.6 & 13.9 & 24.1 \\
\hline 2.0 & 215.4 & 8.6 & 4.0 & 699.5 & 13.9 & 2.0 & 96.2 & 103.7 & 22.5 & 21.8 \\
\hline 4.0 & 351.7 & 14.0 & 4.0 & 590.5 & 15.9 & 2.7 & 8.4 & 163.8 & 29.9 & 18.3 \\
\hline 8.0 & 504.2 & 19.2 & 3.8 & 391.2 & 12.4 & 3.2 & 0 & 218.9 & 31.6 & 14.5 \\
\hline 12.0 & 584.3 & 22.3 & 3.8 & 288.1 & 9.5 & 3.3 & 0 & 241.9 & 31.8 & 13.1 \\
\hline
\end{tabular}


TABLE 3. Average value $(\bar{a})$, std dev $(s)$, and variation coefficient $(v)$ for broadband reflected, total transmitted, and absorbed fluxes at different optical thicknesses. A fractal crystal shape and a solar zenith angle of $\theta=65^{\circ}$ are used.

\begin{tabular}{|c|c|c|c|c|c|c|c|c|c|c|}
\hline \multirow[b]{4}{*}{$\begin{array}{l}\text { Optical } \\
\text { thickness }\end{array}$} & \multicolumn{10}{|c|}{ Fractal $-\theta=65^{\circ}$} \\
\hline & & & & \multicolumn{4}{|c|}{ Transmitted flux } & & & \\
\hline & \multicolumn{3}{|c|}{ Reflected flux } & \multicolumn{2}{|c|}{ Diffuse } & \multicolumn{2}{|c|}{ Direct } & \multicolumn{3}{|c|}{ Absorbed flux } \\
\hline & \multicolumn{2}{|c|}{$\left(\mathrm{W} \mathrm{m}^{-2}\right)$} & $\begin{array}{c}v \\
\%\end{array}$ & \multicolumn{2}{|c|}{$\left(\mathrm{W} \mathrm{m}^{-2}\right)$} & $\begin{array}{c}v \\
\%\end{array}$ & $\begin{array}{c}\bar{a} \\
\left(\mathrm{~W}^{-2}\right)\end{array}$ & \multicolumn{2}{|c|}{$\left(\mathrm{W} \mathrm{m}^{-2}\right)$} & $\begin{array}{c}v \\
\%\end{array}$ \\
\hline 0.5 & 79.9 & 3.0 & 3.8 & 294.0 & 4.0 & 1.4 & 173.3 & 27.6 & 7.0 & 25.4 \\
\hline 1.0 & 132.3 & 5.2 & 3.9 & 341.5 & 6.2 & 1.8 & 53.1 & 48.0 & 11.4 & 23.8 \\
\hline 2.0 & 198.2 & 7.7 & 3.9 & 298.7 & 7.8 & 2.6 & 5.0 & 73.0 & 15.5 & 21.2 \\
\hline 4.0 & 264.3 & 10.0 & 3.8 & 215.8 & 7.1 & 3.3 & 0 & 94.7 & 17.1 & 18.1 \\
\hline 8.0 & 323.4 & 12.0 & 3.7 & 141.8 & 4.6 & 3.2 & 0 & 109.6 & 16.6 & 15.2 \\
\hline 12.0 & 353.0 & 13.2 & 3.7 & 105.5 & 3.5 & 3.3 & 0 & 116.3 & 16.6 & 14.3 \\
\hline
\end{tabular}

dress the effect of cloud structure on the solar radiative fluxes. In a recent study, Buschmann et al. (2002) have investigated the effect of two-dimensional cirrus cloud structure. Their cloud fields have been composed of a sequence of horizontal aircraft measurements. Although the availability of ice particle microphysical properties is an advantage of employing in situ observations, we think that because of sampling and navigation uncertainties the use of 1D aircraft measurements is problematic in reconstructing a real cirrus cloud structure. Therefore we decided to employ 2D volume extinction coefficient data obtained with Raman lidar to study inhomogeneous cirrus clouds. In addition to the advantage of profiling the atmospheric column, Raman lidar measurements of cirrus clouds are available in significantly larger numbers than aircraft observations.

At present, only molecular backscatter lidars [Raman lidar and high spectral resolution lidar; see Ansmann (2002) for a detailed discussion of molecular backscatter lidar techniques] are allowed to measure vertical profiles of volume scattering coefficients that can directly be used as input profiles for radiative transfer calculations. Note that the scattering coefficient equals the extinction coefficient because of the lack of absorption at the ultraviolet or visible Raman lidar wavelengths. Furthermore, for the given large size pa- rameters (ratio of size to wavelength) of ice particles in the solar spectral range the extinction efficiency is very close to 2 so that the lidar extinction profiles can be applied to all other wavelengths in the solar spectrum. In this study three long-duration observations of Arctic cirrus clouds are considered. The cloud systems were measured with the GKSS Research Center Raman lidar (Reichardt et al. 1996) above the Swedish research facility Esrange $\left(67.9^{\circ} \mathrm{N}, 21.1^{\circ} \mathrm{E}\right)$ in January 1997, for height versus time displays of the cirrus backscatter coefficient see Reichardt et al. (2002). Data were acquired with 120 -s temporal and 120-m spatial resolution. For the determination of the extinction coefficient profiles (569 profiles in total) $20 \mathrm{~min}$ of consecutive lidar data were integrated and the molecular nitrogen Raman signal was smoothed with a sliding average window length of $600 \mathrm{~m}$ in order to reduce statistical errors. As an example, Fig. 4 (bottom) shows the second volume extinction field obtained on 8 January 1997 together with the time series of the optical thickness. Besides data integration time, the horizontal resolution depends on wind speed at which the cirrus is advected over the lidar. Here, we use National Centers for Environmental Prediction-National Center for Atmospheric Research (NCEP-NCAR) reanalysis data (Kistler et al. 2001) to obtain the horizontal scale of each time series. For the time series shown in Fig. 4, the

TABLE 4. Average value $(\bar{a})$, std dev $(s)$, and variation coefficient $(v)$ for broadband reflected, total transmitted, and absorbed fluxes at different optical thickness. A columnar hexagonal crystal shape and a solar zenith angle of $\theta=35^{\circ}$ are used.

\begin{tabular}{|c|c|c|c|c|c|c|c|c|c|c|}
\hline \multirow[b]{4}{*}{$\begin{array}{l}\text { Optical } \\
\text { thickness }\end{array}$} & \multicolumn{10}{|c|}{ Column $-\theta=35^{\circ}$} \\
\hline & & & & \multicolumn{4}{|c|}{ Transmitted flux } & & & \\
\hline & \multicolumn{3}{|c|}{ Reflected flux } & \multicolumn{2}{|c|}{ Diffuse } & \multicolumn{2}{|c|}{ Direct } & \multicolumn{3}{|c|}{ Absorbed flux } \\
\hline & \multicolumn{2}{|c|}{$\left(\mathrm{W} \mathrm{m}^{-2}\right)$} & $\begin{array}{c}v \\
\%\end{array}$ & \multicolumn{2}{|c|}{$\left(\mathrm{W} \mathrm{m}^{-2}\right)$} & $\begin{array}{c}v \\
\%\end{array}$ & $\left(\begin{array}{c}\bar{a} \\
\left(\mathrm{~W} \mathrm{~m}^{-2}\right)\end{array}\right.$ & \multicolumn{2}{|c|}{$\left(\mathrm{W} \mathrm{m}^{-2}\right)$} & $\begin{array}{c}v \\
\%\end{array}$ \\
\hline 0.5 & 46.4 & 4.3 & 9.3 & 434.3 & 2.6 & 0.6 & 600.3 & 33.3 & 6.8 & 20.4 \\
\hline 1.0 & 85.7 & 8.1 & 9.4 & 639.5 & 4.1 & 0.6 & 326.1 & 63.0 & 12.1 & 19.2 \\
\hline 2.0 & 156.2 & 14.1 & 9.0 & 750.3 & 5.2 & 0.7 & 96.2 & 111.6 & 19.4 & 17.1 \\
\hline 4.0 & 268.0 & 21.3 & 7.9 & 663.0 & 4.1 & 0.6 & 8.4 & 175.0 & 24.9 & 14.2 \\
\hline 8.0 & 411.0 & 27.3 & 6.7 & 470.7 & 2.6 & 0.6 & 0 & 232.5 & 26.2 & 11.3 \\
\hline 12.0 & 495.1 & 29.8 & 6.0 & 362.0 & 3.3 & 0.9 & 0 & 257.2 & 26.9 & 10.9 \\
\hline
\end{tabular}


TABLE 5. Average value $(\bar{a})$, std dev $(s)$, and variation coefficient $(v)$ for broadband reflected, total transmitted, and absorbed fluxes at different optical thicknesses. A columnar hexagonal crystal shape and a solar zenith angle of $\theta=65^{\circ}$ are assumed.

\begin{tabular}{|c|c|c|c|c|c|c|c|c|c|c|}
\hline \multirow[b]{4}{*}{$\begin{array}{l}\text { Optical } \\
\text { thickness }\end{array}$} & \multicolumn{10}{|c|}{ Column $-\theta=65^{\circ}$} \\
\hline & & & & \multicolumn{4}{|c|}{ Transmitted flux } & & & \\
\hline & \multicolumn{3}{|c|}{ Reflected flux } & \multicolumn{2}{|c|}{ Diffuse } & \multicolumn{2}{|c|}{ Direct } & \multicolumn{3}{|c|}{ Absorbed flux } \\
\hline & \multicolumn{2}{|c|}{$\left(\mathrm{W} \mathrm{m}^{-2}\right)$} & $\begin{array}{c}v \\
\%\end{array}$ & \multicolumn{2}{|c|}{$\left(\mathrm{W} \mathrm{m}^{-2}\right)$} & $\begin{array}{c}v \\
\%\end{array}$ & $\begin{array}{c}\bar{a} \\
\left(\mathrm{~W} \mathrm{~m}^{-2}\right)\end{array}$ & \multicolumn{2}{|c|}{$\left(\mathrm{W} \mathrm{m}^{-2}\right)$} & $\begin{array}{c}v \\
\%\end{array}$ \\
\hline 0.5 & 60.2 & 4.3 & 7.1 & 310.4 & 2.0 & 0.6 & 173.3 & 30.9 & 6.2 & 20.1 \\
\hline 1.0 & 102.7 & 7.1 & 6.9 & 365.6 & 3.0 & 0.8 & 53.1 & 53.5 & 10.0 & 18.7 \\
\hline 2.0 & 160.9 & 10.2 & 6.3 & 327.9 & 3.3 & 1.0 & 5.0 & 81.1 & 13.5 & 16.6 \\
\hline 4.0 & 225.1 & 12.7 & 5.6 & 245.3 & 2.1 & 0.9 & 0 & 104.5 & 14.6 & 14.0 \\
\hline 8.0 & 285.1 & 14.5 & 5.1 & 170.1 & 0.9 & 0.5 & 0 & 120.0 & 14.2 & 11.9 \\
\hline 12.0 & 316.6 & 15.4 & 4.9 & 131.6 & 1.0 & 0.8 & 0 & 126.7 & 14.5 & 11.4 \\
\hline
\end{tabular}

horizontal/vertical resolution is $2500 / 120 \mathrm{~m}$. To attribute a phase function and single scattering albedo to each cloud cell, we determined the relation between extinction and effective radius from the 114 size distributions measured during the EUCREX campaign (Fig. 5) and then used the functional dependence to map the lidar-observed volume extinction coefficients onto effective radii. We employ this relation to obtain estimates of effective radius although our analysis and the results of previous studies [see, e.g., Macke et al. (1998), their Fig. 5 yields a correlation coefficient of 0.41$]$ indicate that the correlation between extinction and effective radius is relatively weak. As it is described below, we designed our model simulations so that the significant uncertainty in this correlation is taken into account.

The following two cloud scenarios are studied:

1) Inhomogeneous (2D): $2 \mathrm{D}$ extinction coefficient field, and 2D phase function and single scattering albedo fields. The original lidar time series are taken as the $2 \mathrm{D}$ extinction fields. For each measured extinction coefficient, a mean effective radius and its standard deviation is selected according to the regression line shown in Fig. 5. Both quantities are used to completely characterize a Gaussian probability density distribution (PDF). A random number weighted by this PDF is chosen as the actual effective radius for this extinction coefficient This approach results in a $2 \mathrm{D}$ field for phase function and single scattering albedo, which is statistically in accordance with the extinction coefficient-to-effective radius relation. The cloud field is assumed to be homogeneous along the third dimension.

2) Plane parallel (0D): A plane-parallel homogeneous cloud specified by an averaged phase function and an averaged single scattering albedo. The optical thickness is equal to the mean optical thickness of the original lidar time series.

The Monte Carlo radiative transfer calculation for these scenarios has been performed for a solar zenith angle of $35^{\circ}$ and with periodic boundary conditions along the horizontal direction. The solar azimuth angle is chosen so that the sun is shining in positive direction of the horizontal axis, that is, from left to right in Fig. 4.

Figure 4 (top) shows the solar broadband radiative fluxes normalized to the incoming flux at each vertical cloud column for the 2D case together with the lidar cloud structure. Also shown is the net horizontal transport with white and dark bars denoting a net energy gain and loss. By definition, the net horizontal transport is zero everywhere for the plane-parallel case. In the 2D situation, large net horizontal transports result from strong horizontal gradients in the optical thickness. For the cirrus clouds studied here, however, net horizontal transport is close to zero most of the time. The domainaveraged normalized fluxes of the $2 \mathrm{D}$ case and the $0 \mathrm{D}$ case are compared in Fig. 6. In accordance with numerous previous studies [mostly for water clouds, though, see e.g., O'Hirok and Gautier (1998) and references therein], an inhomogeneous cloud structure leads to larger direct transmittance and less reflectance in most cases. Diffuse transmittance as well as absorptance are slightly smaller in the 2D case. However, note that the sign of the cloud structure effect on diffuse transmittance and absorptance depends on the solar zenith angle. Therefore, we expect to get different $2 \mathrm{D}$ effects for these two fluxes at other solar zenith angles. The differences in the solar radiative fluxes that are due to the different particle geometries are comparable to the differences between the results obtained for homogeneous and structured clouds for the diffuse transmittance and for the absorptance. Only for the reflected flux, the shape dependency is more strongly pronounced. Combining these findings with our results reported in section 2, we conclude that ice crystal shape appears to be the second most important parameter for cirrus radiative transfer after cloud optical thickness.

We briefly mention two further comparisons without visualizing the results. First, keeping the microphysical properties constant over the entire cloud domain in the 2D case only leads to small differences in the radiative fluxes compared to the full 2D inhomogeneity. Therefore, it appears that the spatial variability of the cirrus microphysical properties only plays a minor role. Sec- 

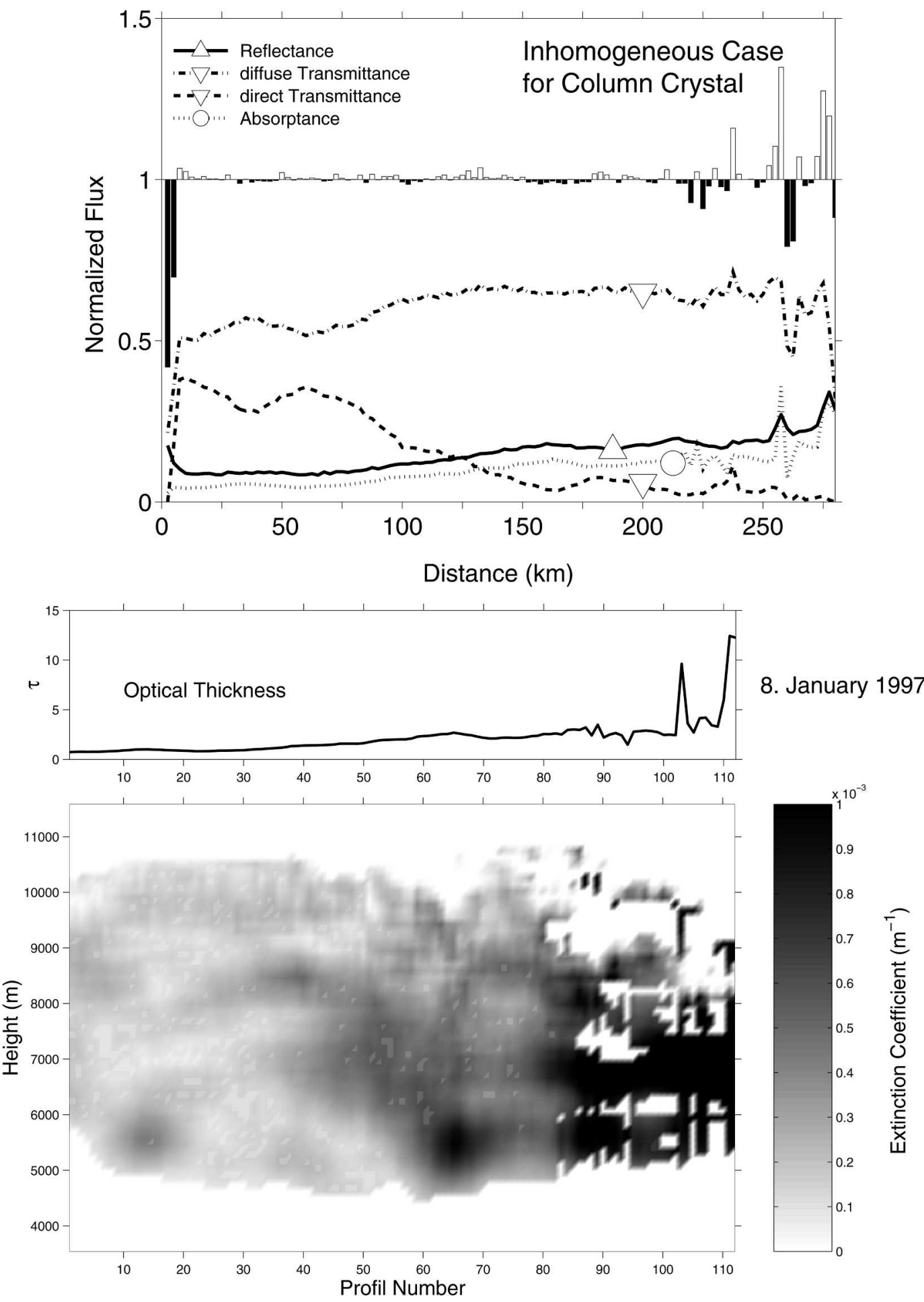

FIG. 4. (top) Normalized solar broadband fluxes for the inhomogeneous cirrus case (8 Jan 1997 data). Open and solid bars indicate net energy gain and loss due to net horizontal transport, respectively. (bottom) Height vs time display of extinction coefficient from Raman lidar measurements above Esrange (Sweden) on 8 Jan 1997. The measurement wavelength is $355 \mathrm{~nm}$. Note that for a better illustration, the extinction coefficients larger than $10^{-3}$ $\mathrm{m}^{-1}$ have the same color code. 


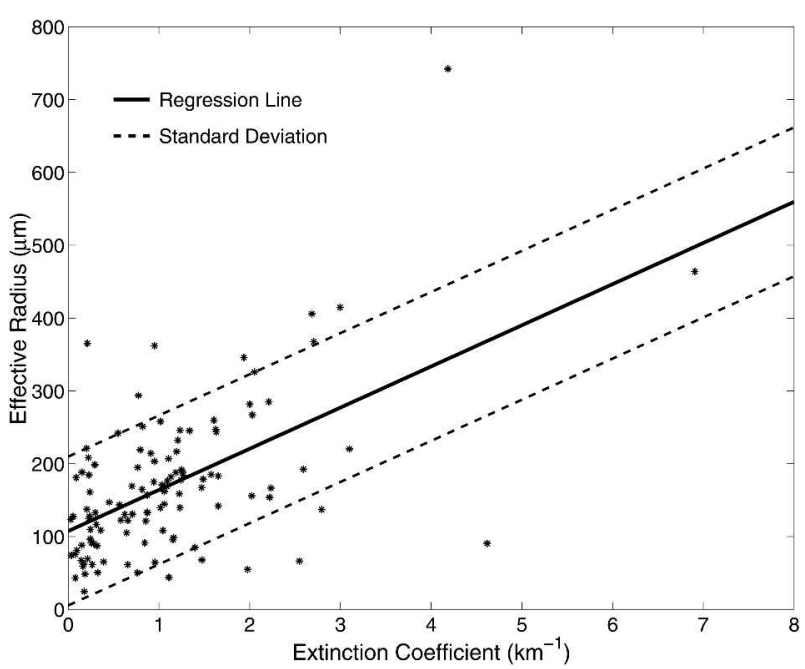

FIG. 5. Correlation between effective radius and extinction coefficient as determined from cirrus size distributions measured during the EUCREX campaign.

ond, we calculated the radiative fluxes for each lidar column in independent column mode (plane parallel) and found small but significant differences in the planeparallel case. Thus, the vertical structure of the cirrus alone has a significant effect on the solar radiative fluxes. Smaller and thus more reflective ice particles near the cloud top lead to larger reflectivities, smaller transmissivities and slightly larger absorptivities.

\section{Summary and conclusions}

In this paper the influence of microphysical and macrophysical characteristics of cirrus clouds on the solar radiative fluxes was examined. The spectral solar radiation transport calculations were accomplished with the radiation transport model GRIMALDI. In the first part of the work, with the use of two crystal shapes and a large number of different crystal size distributions, the variability of microphysical properties in cirrus clouds and its effect on the spectral and broadband radiation balance are evaluated.

The broadband radiative fluxes show an explicit dependence on the size distributions. The largest variation coefficients are found for the broadband absorbed flux. Upward and downward broadband fluxes show considerably smaller variability. The variation coefficients for both crystal shapes are below $10 \%$. In some wavelength bands, very large variabilities arise. However, these large variability coefficients have only little effect if broadband fluxes are considered because of their small weight in the entire solar spectrum. Furthermore, the choice of the crystal shape has a stronger influence on the reflection and transmission characteristics of radiation than the variability in the size distribution.

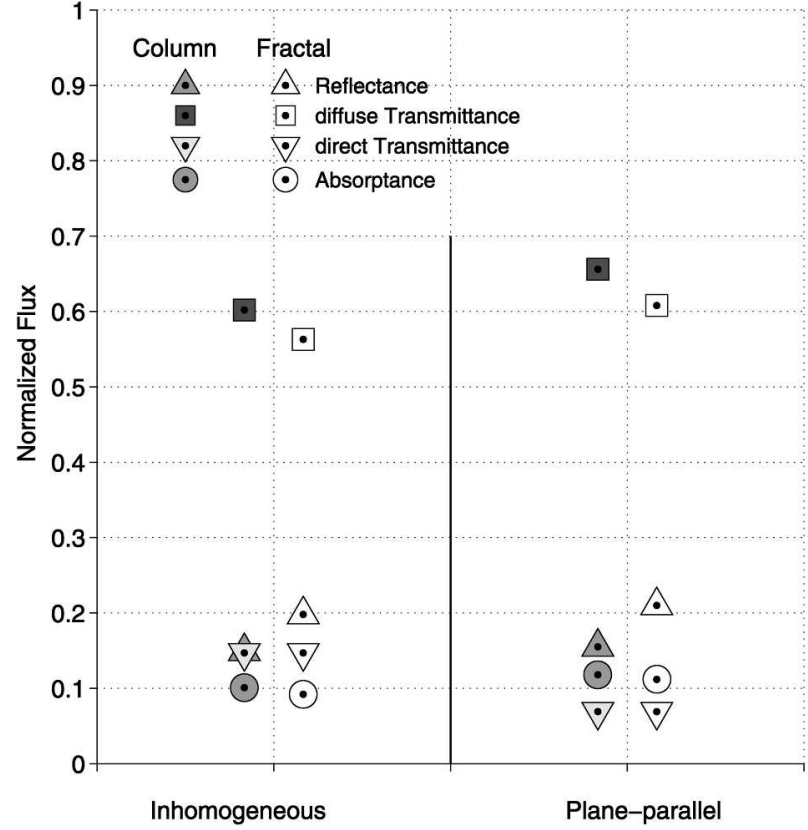

FIG. 6. Domain-averaged normalized broadband fluxes derived for 2D inhomogeneous, and plane-parallel cirrus clouds.

To examine the influence of cloud inhomogeneity on the radiation balance in cirrus clouds, we used extinction profiles measured with Raman lidar as input data for the radiation transport model. In addition, particle scattering and absorption properties were chosen randomly for each grid cell. It was found that the spatial variability of the extinction coefficient leads to significant differences in the solar fluxes compared to the commonly used approximation of plane-parallel homogeneous clouds. The spatial variability of particle size has little additional effects. The differences in the solar radiative fluxes that are due to the different particle geometries are comparable to the differences between the results obtained for homogeneous and structured clouds for the diffuse transmittance and for the absorptance. Only for the reflected flux is the shape dependency more strongly pronounced.

Since the results of this sensitivity study are based on data from one cirrus field campaign in the midlatitudes and from one time series of Raman lidar measurements in near-polar regions, our quantitative findings do not apply globally. Still, because we used real input dataexcept for ice particle shape-for the cirrus cases, our results can be regarded as a first estimate of the effect of cirrus microphysical variability on the solar radiative fluxes. Future work will include larger datasets with emphasis on different climatological/synoptical situations.

Given the uncertainties in cirrus radiative fluxes as discussed in the present paper it is clear that additional information on ice particle shape and size distributions as well as on small-scale cloud structure will substan- 
tially improve our ability to calculate the solar radiative fluxes of this cloud type. Global climatologies of particle effective radius from various remote sensing techniques (e.g., Rädel et al. 2003) may provide first guesses of effective ice crystal sizes for specific climatic regions, for specific weather conditions, and, most importantly, for specific stages of the cloud development.

It has been demonstrated theoretically (e.g., Macke et al. 1996) and experimentally (e.g., DoutriauxBoucher et al. 2000) that the polarized reflected solar radiation contains information on ice particle habit. Therefore, future satellite radiometers that are capable of measuring polarization may help to provide similar climatologies of dominant particle geometries.

The structure of cirrus clouds is closely linked to the atmospheric and to the internal cloud dynamics with strong turbulent patterns. See also the relevant chapters in Lynch et al. (2002). The analysis of large lidar and radar (and combined lidar/radar) datasets may provide stochastic information on small-scale variabilities of cirrus extinction coefficient along the vertical and the horizontal direction.

Acknowledgments. We are grateful to Ronald Scheirer for providing the radiative transfer model GRIMALDI and helpful assistance.

\section{REFERENCES}

Ansmann, A., 2002: Molecular-backscatter lidar profiling of the volume-scattering coefficient in cirrus. Cirrus, D. K. Lynch et al., Eds., Oxford University Press, 197-210.

Buschmann, N., G. McFarquhar, and A. Heymsfield, 2002: Effects of observed horizontal inhomogeneities within cirrus clouds on solar radiative transfer. J. Geophys. Res., 107, 4445, doi:10.1029/2001JD001273.

Chen, T., W. B. Rossow, and Y. Zhang, 2000: Radiative effects of cloud-type variations. J. Climate, 13, 264-286.

Doutriaux-Boucher, M., J.-C. Buriez, G. Brogniez, L. Labonnote, and A. Baran, 2000: Sensitivity of retrieved POLDER directional cloud optical thickness to various ice particle models. Geophys. Res. Lett., 27, 109-112.

Francis, P. N., 1995: Some aircraft observations of the scattering properties of ice crystals. J. Atmos. Sci., 52, 1142-1154.

Hansen, J. E., and L. D. Travis, 1974: Light scattering in planetary atmospheres. Space Sci. Rev., 16, 527-610.

Houghton, J. T., Y. Ding, D. J. Griggs, M. Nouger, P. J. van der Linden, X. Dai, K. Maskell, and D. Xiaosu, 2001: Climate Change 2001: The Science of Climate Change. Cambridge University Press, $881 \mathrm{pp}$.

Kinne, S., and Coauthors, 1997: Cirrus cloud radiative and microphysical properties from ground observations and in situ measurements during FIRE 1991 and their application to exhibit problems in cirrus solar radiative transfer modeling. $J$. Atmos. Sci., 54, 2320-2344.
Kistler, R., and Coauthors, 2001: The NCEP-NCAR 50-year reanalysis: Monthly means CD-ROM and documentation. Bull. Amer. Meteor. Soc., 82, 247-267.

Korolev, A. V., G. A. Isaac, I. P. Mazin, and H. W. Barker, 2001: Microphysical properties of continental clouds from in situ measurements. Quart. J. Roy. Meteor. Soc., 127, 2117-2151.

Lynch, D. K., K. Sassen, D. O. Starr, and G. Stephens, 2002: Cirrus. Oxford University Press, $480 \mathrm{pp}$.

Macke, A., J. Mueller, and E. Raschke, 1996: Single scattering properties of atmospheric ice crystals. J. Atmos. Sci., 53, 2813-2825.

_ , P. N. Francis, G. M. McFarquar, and S. Kinne, 1998: The role of ice particle shapes and size distributions in the single scattering properties of cirrus clouds. J. Atmos. Sci., 55, 2874 2883.

_ D. Mitchell, and L. von Bremen, 1999: Monte Carlo radiative transfer calculations for inhomogeneous mixed phase clouds. Phys. Chem. Earth, 24, 237-241.

Magano, C., and C. W. Lee, 1966: Meteorological classification of natural snow crystals. J. Fac. Sci. Hokkaido Univ. Series VII, 2, 321-335.

Mishchenko, M. I., L. D. Travis, and A. Macke, 2000: T-matrix method and its applications. Light Scattering by Nonspherical Particles, M. Mishchenko, J. W. Hovenier, and L. Travis, Eds., Academic Press, 147-172.

O'Hirok, W., and C. Gautier, 1998: A three-dimensional radiative transfer model to investigate the solar radiation within cloudy atmospheres. Part I: Spatial effects. J. Atmos. Sci., 55, 21622179.

Poetzsch-Heffter, C., Q. Liu, E. Ruprecht, and C. Simmer, 1995: Effect of cloud types on the earth radiation budget calculated with the ISCCP C1 dataset: Methodology and initial results. J. Climate, 8, 829-843.

Rädel, G., C. Stubenrauch, R. Holz, and D. Mitchell, 2003: Retrieval of effective ice crystal size in the infrared: Sensitivity study and global measurements from TIROS-N operational vertical sounder. J. Geophys. Res., 108, 4281, doi:10.1029/ 2002JD002801.

Raschke, E., and Coauthors, 1997: European Cloud and Radiation Experiment (EUCREX)-Final Report on the Project EV5V-CT 92-0130 EUCREX-2. GKSS-Forschungszentrum Geesthacht GmbH, 154 pp.

Reichardt, J., U. Wandinger, M. Serwazi, and C. Wetkamp, 1996: Combined raman lidar for aerosol, ozone, and moisture measurements. Opt. Eng., 35, 1457-1465.

_ S. Reichardt, A. Behrendt, and T. J. McGee, 2002: Correlations among the optical properties of cirrus-cloud particles: Implications for spaceborne remote sensing. Geophys. Res. Lett., 29, 1668, doi:10.1029/2002GL014836.

Scheirer, R., and A. Macke, 2001: On the accuracy of the independent column approximation in calculating the downward fluxes in the UV-A, UV-B and PAR spectral ranges. J. Geophys. Res., 106, 14 301-14 312.

, and _ 2003: Cloud-inhomogeneity and broadband solar fluxes. J. Geophys. Res., 108, 4599, doi:10.1029/2002JD003321.

Thekaekara, M. P., 1974: Extraterrestrial solar spectrum 3000$6000 \AA$ at $1-\AA ̊$ intervals. Appl. Opt., 13, 518-522.

Zhang, Y., A. Macke, and F. Albers, 1999: Effect of crystal size spectrum and crystal shape on stratiform cirrus radiative forcing. J. Atmos. Res., 52, 59-75. 